\title{
Chromatin in situ proximity (ChrISP): Single-cell analysis of chromatin proximities at a high resolution
}

Xingqi Chen ${ }^{1,{ }^{*}}$, Chengxi Shi ${ }^{1,{ }^{*}}$, Samer Yammine ${ }^{1,{ }^{*}}$, Anita Göndör ${ }^{1}$, Daniel Rönnlund ${ }^{2}$, Alejandro FernandezWoodbridge ${ }^{1}$, Noriyuki Sumida $^{1}$, Jerker Widengren ${ }^{2}$, and Rolf Ohlsson ${ }^{1}$

${ }^{1}$ Department of Microbiology, Tumor and Cell Biology, Karolinska Institutet, Stockholm, Sweden and ${ }^{2}$ Applied

Physics, AlbaNova University Center, Royal Institute of Technology, Sweden

${ }^{*}$ X.C., C.S., and S.Y. contributed equally to this work

BioTechniques 56:117-124 (March 2014) doi 10.2144/000114145

Keywords: chromosome; epigenetics; conformation

Supplementary material for this article is available at www.BioTechniques.com/article/114145.

Current techniques for analyzing chromatin structures are hampered by either poor resolution at the individual cell level or the need for a large number of cells to obtain higher resolution. This is a major problem as it hampers our understanding of chromatin conformation in single cells and how these respond to environmental cues. Here we describe a new method, chromatin in situ proximity (ChrISP), which reproducibly scores for proximities between two different chromatin fibers in 3-D with a resolution of $\sim 170 \AA$ in single cells. The technique is based on the in situ proximity ligation assay (ISPLA), but ChrISP omits the rolling circle amplification step (RCA). Instead, the proximities between chromatin fibers are visualized by a fluorescent connector oligonucleotide DNA, here termed splinter, forming a circular DNA with another circle-forming oligonucleotide, here termed backbone, upon ligation. In contrast to the regular ISPLA technique, our modification enables detection of chromatin fiber proximities independent of steric hindrances from nuclear structures. We use this method to identify higher order structures of individual chromosomes in relation to structural hallmarks of interphase nuclei and beyond the resolution of the light microscope.

Higher order chromatin conformations result from packaging of the genome within the confines of the nucleus. This packaging must not only allow for the regulated accessibility of trans-acting factors to generate local chromatin loops but also enable the anchoring of the chromatin to structural hallmarks $(1,2)$ to facilitate the emergence of robust phenotypes.

Our understanding of chromatin conformations and how these relate to hallmarks of nuclear structures is currently based on a combination of confocal DNA fluorescence in situ hybridization (DNA FISH) and the so-called $\mathrm{C}$ techniques $(3 \mathrm{C}, 4 \mathrm{C}, 5 \mathrm{C}$, and $\mathrm{Hi}-\mathrm{C})(2-4)$. The latter methods, which are based on the in situ ligation of cross-linked chromatin fibers to assess chromatin fiber proximities in a highthroughput manner, can have a very high resolution defined by the formaldehyde crosslinking agent (4). However, these methods do not robustly address the frequency of such features within a cell population, complicating the interpretation of chromatin conformation during complex biological processes. Conversely, single-cell studies of chromatin proximities using DNA FISH are severely hampered by that technique's low 3-D resolution (5).

Although the lateral resolution of a confocal microscope is limited to $\sim 250$ $\mathrm{nm}$ by diffraction, this resolution can be considerably increased in a stimulated emission depletion (STED) microscope (6). However, in many realizations of STED microscopy, the axial resolution is comparable to that of confocal microscopy. Even though recent developments in far field optical super resolution microscopy now allows the resolution in all three planes to be improved by about an order of magnitude, i.e. from about 1 $\mu \mathrm{m}$ down to approximately $100 \mathrm{~nm}(7,8)$, this is still is not sufficient to discern different types of chromatin structures.

Given that complex biological processes, such as those represented by dynamic changes in chromatin structure, cannot be understood unless they are examined in single cells (9), there is a

\section{Method summary:}

Combining features of the in situ proximity ligation technique with conventional DNA FISH/immunostaining, the method enables the identification of the proximity of two different locations in chromatin, such as different chromatin regions or a chromatin region and a chromatin mark, in relation to structural hallmarks of the nucleus. 
strong need for a new method that has the pros of $3 \mathrm{C}$ (or other $\mathrm{C}$ techniques) and DNA FISH technologies while minimizing their cons. Although a single-cell $\mathrm{Hi}-\mathrm{C}$ method has recently been described, the resolution of chromatin proximities was very poor (10).

This study describes the ChrISP method, which not only explores chromatin fibre proximities beyond the current resolution of the microscope, but also allows an analysis of the frequencies of such features in cell populations. The new method is based on the analysis of proximities between chromatin fibres brought together in the context of higher order chromatin structures (Figure 1A) with a resolution that goes far beyond that of a conventional confocal microscope. It exploits features of the in situ proximity ligation assay (ISPLA) (11), but without the rolling circle amplification (RCA) step. This modification neutralizes steric hindrances provided by nuclear substructures and provides a continuum of signals rather than large dots typical of the rolling circle amplification step. DNA probes labeled with either digoxigenin or biotin are hybridized to formaldehyde-fixed cells followed by primary antibody detection. After the primary antibodies are bound by secondary antibodies conjugated to different oligonucleotide DNA sequences, backbone (long connector) and fluorescently labeled splinter (short connector) oligonucleotides are added. If two different secondary antibodies are within $170 \AA$ of each other, their respective conjugated oligonucleotides are close enough to allow the backbone and splinter oligonucleotides to simultaneously anneal to them, and these can then be ligated to each other to form a fluorescently labeled circular DNA molecule. The signal derived from the labeled splinter oligonucleotide will only survive subsequent washing if it has been ligated into the circular DNA. ChrISP represents a major improvement over existing techniques in terms of resolution at the light microscopic level and with a versatility that makes it suitable for numerous applications focusing on chromatin structures and associated marks as well as how these relate to nuclear hallmarks.

\section{Materials and methods}

\section{Cell culture and preparation}

Human colon cancer cells (HCT116) were seeded for $48 \mathrm{~h}$ on an 8 -well Lab-Tek-II Chamber Slide system (154534; Thermo Scientific, Waltham, MA), then fixed with $3 \%$ paraformaldehyde (PFA) for $15 \mathrm{~min}$ at room temperature (4), and then stored in $70 \%$ ethanol at $-20^{\circ} \mathrm{C}$ until use.

\section{DNA probes and FISH protocol}

Human Cot-1 DNA (15279-011; Invitrogen, Carlsbad, CA) was labeled with Biotin-16-dUTP (11093070910; Roche Applied Science, Penzberg, Germany) or Digoxigenin-11-dUTP (11573152910; Roche Applied Science) using the Bioprime Array CGH kit (18095-011; Invitrogen). Chromosome 11 painting probe (a kind gift from Irina Solovei) was labeled as previously described (12).

DNA FISH was performed as previously described (13). In brief, the cross- linked cells were rehydrated in PBS without air drying, permeabilized with $0.5 \%$ Triton X-100 in PBS, and the genomic DNA denatured in $2 \times$ SSC $/ 50 \%$ formamide at $80^{\circ} \mathrm{C}$ for $40 \mathrm{~min}$.

HCT116 DNA fibers were combed on CombiCoverslips (COV-001; Genomic Vision, Paris, France) with the Molecular combing system as previously described (14). DNA fibers were denatured in 0.5 $\mathrm{M} \mathrm{NaOH} / 1 \mathrm{M} \mathrm{NaCl}$ and then hybridized with human Cot-1 probes.

The hybridization with biotin- and digoxigenin-labeled DNA probes was performed overnight at $37^{\circ} \mathrm{C}$ in $2 \times \mathrm{SSC}$, $50 \%$ formamide, and $12 \%$ dextran sulfate. Cells/DNA fibers were washed twice with $2 \times$ SSC $/ 50 \%$ formamide for $15 \mathrm{~min}$ at $40^{\circ} \mathrm{C}$, twice with $2 \times$ SSC for $15 \mathrm{~min}$ at $40^{\circ} \mathrm{C}$, and then twice with $0.1 \times$ SSC for $15 \mathrm{~min}$ at $60^{\circ} \mathrm{C}$. The visualization of the biotin and digoxigenin marks was performed by first blocking with antibody diluent (00-3218; Invitrogen) at room temperature for $1 \mathrm{~h}$, followed by incubation with primary antibodies (mouse anti-Digoxigenin, 11333062910; Roche Applied Science)(rabbit antiBiotin, Ab53494; Abcam, Cambridge, $\mathrm{UK}$ ) overnight at $4^{\circ} \mathrm{C}$. After washing $5 \times$ 10 min with $0.05 \%$ Tween 20 in PBS, the fixed cells were incubated with secondary fluorescent antibodies (goat anti-rabbit IgG Dylight 649, 84545; Thermo Scientific)(goat anti-mouse IgG Dylight 594, 35511; Thermo Scientific) for biotin and digoxigenin detection at $37^{\circ} \mathrm{C}$ for 1 $h$, followed by $6 \times 15 \mathrm{~min}$ washes with $0.05 \%$ Tween 20 in PBS at room temperature. DNA was counterstained and cells were mounted in Vectashield mounting medium with DAPI (H-1200; Vector
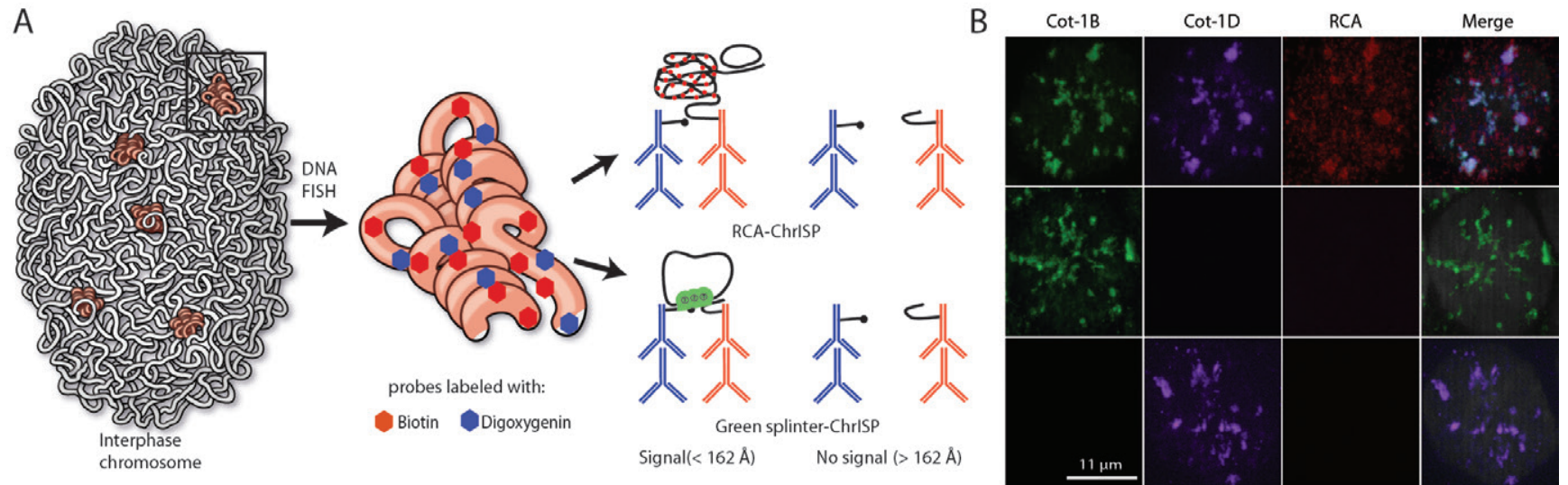

Figure 1. The ChrISP method and proof-of-principle. (A) Schematic illustration of the ChrISP method and the two strategies used to detect proximal chromatin domains both in cis and in trans using splinter oligonucleotide and backbone oligonucleotide ligation; RCA: rolling circle amplification. Green splinter-ChrISP (bottom right of panel A) depicts an alternative strategy using a fluorescently labeled splinter oligonucleotide. (B) ChrISP analysis of proximities between repeat elements. Proximities were detected by using biotin/digoxigenin-labeled Cot-1 probes followed by RCA in formaldehyde crosslinked nuclei of HCT116 cells, as schematically shown in panel (A). As negative controls for ChrlSP analysis, either biotin- (lowest row) or digoxigenin- (mid-row) labeled probe was omitted. 

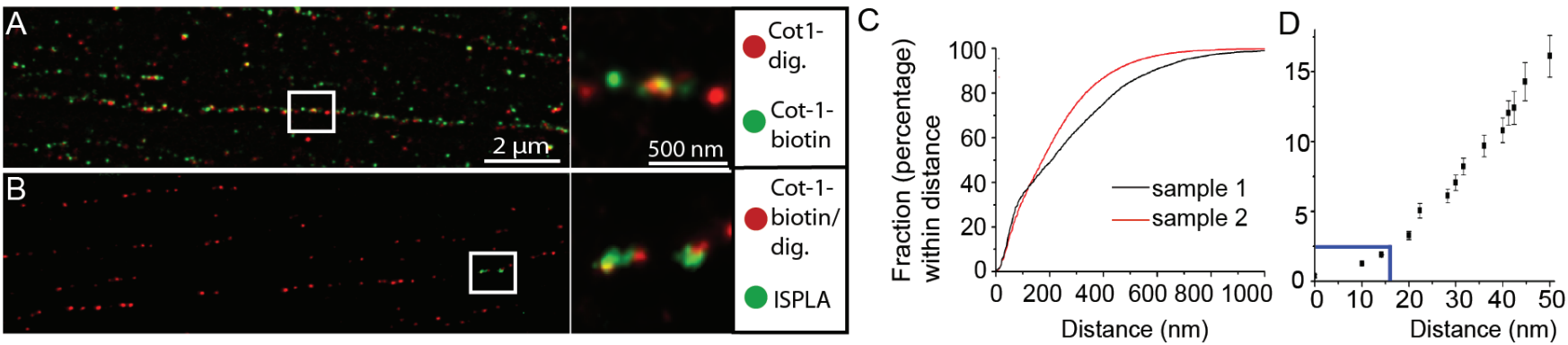

Figure 2. Resolution of the ChrISP method. (A) STED microscopy images showing fiber FISH analysis of Cot-1 DNA distribution with the biotin and digoxigenin probes shown in green and red, respectively. (B) RCA products (green) were visualized in a STED microscope, with both biotin and digoxigenin probes shown in red. In both $A$ and $B$, the right panels explain the color code, and the middle panels show enlarged portions of the regions in the white square in the left panels. (C) Cumulative curves from two independent analyses (red and black lines) measuring the center-to-center distances between biotin and digoxigenin-marked regions. (D) Enlargement of the cumulative curves shown in (C). Rolling circle amplification (RCA) frequency on DNA fibers was $2.46 \%$, corresponding to a resolution of $16.2 \mathrm{~nm}$; the relationship between RCA frequency and resolution is indicated by the blue lines.

Labs, Burlingame, CA). Cell imaging was carried out on a Leica microscope (Leica Microsystems AB, Kista, Sweden) with an OptiGrid device (Quorum Angstrom Grid Confocal System; Quorum Technologies Inc., Guelph, Ontario, Canada.) and analyzed with Volocity software (Improvision, Perkin Elmer, Waltham, MA).

ChrISP analysis

Modified $F\left(a b^{\prime}\right)$, fragments: The proximity probes were covalently linked via their $5^{\prime}$ ends to $F\left(a b^{\prime}\right)$, fragments (SoluLink, San Diego, CA). F(ab') fragment donkey anti-mouse IgG (715-006-150; Jackson ImmunoResearch, West Grove, PA) was linked to the non-priming amine-modified oligonucleotide $\mathrm{NH}_{2}-\mathrm{AAAAAAAA}$ A AGACGCTAATAGTTAAGACG CTT[UUU] (the sequence within the brackets is 2'O-methyl-RNA) (Trilink BioTechnologies, San Diego, CA).

$\left.\mathrm{F}(\mathrm{ab})^{\prime}\right)$ fragment donkey anti-rabbit IgG (711-006-152; Jackson ImmunoResearch) was linked to the RCA priming amine-modified oligonucleotide $\mathrm{NH}_{2}$ - A A A A A A A A A TATGACAGAACTAGACACTCTT (Trilink BioTechnologies).

Fluorescently labeled splinter oligonucleotide: A modified splinter oligonucleotide was designed by adding green fluorescent nucleotides (fluoresceinconjugated deoxythymidine; FlcdT) to the nonhybridizing middle part of the construct to avoid any mis-annealing or alterations in the ISPLA system ([Phos] GTTCTGTCATA[FlcdT][FlcdT] [FlcdT]AAGCGTCTTAA) (SigmaAldrich, St. Louis, MO).

Cot-1 RCA ChrISP: Cot-1 DNA FISH was done as described above with the exception that the dextran sulfate in the hybridization buffer was replaced with dextran (buffer composition: $2 \times$ SSC, $50 \%$ formamide, $12 \%$ dextran). The reason for the change in buffer was that the presence of dextran sulfate inhibited the RCA step (data not shown). Following incubation with antibodies and washing, ISPLA was performed using the Duolink detection kit (90103; Olink Bioscience, Uppsala, Sweden).

Chromosome 11 territory/Cot-1 ChrISP: Splinter and backbone oligonucleotide hybridization and ligation were done as previously described (11) using the FlcdT-labeled splinter oligonucleotide. To remove splinter oligonucleotides annealing to either priming or non-priming antibodies, while preserving the ligated circular DNA indicating the proximity of priming and non-priming antibodies, an additional overnight washing with $0.1 \times$ SSC at $37^{\circ} \mathrm{C}$ was added at the end.

\section{STED microscopy}

STED images were acquired using a custom built microscope $(6,15)$. In brief, a single supercontinuum laser (SC-450-PP-HE; Fianium Ltd., Southampton, $\mathrm{UK}$ ) was used for the selection of 2 excitation beams $(570 \pm 5$ and 647 $\pm 5 \mathrm{~nm})$ and 2 STED beams $(710 \pm 10$ and $750 \pm 10 \mathrm{~nm}$ ). In order to create the doughnut shaped focus of the two STED beams, the beams were passed through separate vortex phase plates (VPP-1; RPC Photonics, Rochester, NY) prior to being sent to the objective. The doughnut shape of the STED beam only allowed fluorophores in the very center of the focus to emit fluorescence, since the fluorophores outside the center were affected by the STED intensity and, if excited, would be switched off by stimulated emission. This effectively increased the resolution, and the acquired resolution in this system was $40 \mathrm{~nm}$ for both channels. Both selected dyes have fluorescence lifetimes below $4 \mathrm{~ns}$, so by separating the excitation and corresponding STED beams of both dyes by $40 \mathrm{~ns}$, the crosstalk between the dyes could be efficiently reduced to only a few percent. The same image settings were used for all images, with an image size of $15 \times 10 \mu \mathrm{m}^{2}$, pixel size of $20 \mathrm{~nm}$, and pixel dwell time of $1 \mathrm{~ms}$.

STED image analysis of Cot-1 DNA FISH and ChrISP resolution calculation Before image analysis, STED images were rescaled to $10 \mathrm{~nm}$ pixels and then deconvoluted using 10 iterations of the Richardsson-Lucy deconvolution algorithm with a $40 \mathrm{~nm}$ Lorentzian point spread function (PSF)(16). Image analysis was performed using custom-written code in Matlab (MathWorks Inc, Natick, Massachusetts) where the centers of the labeled probes were determined as the locations of their respective fluorescence peaks. The distance between the labeled Cot-1 probes was then determined by a nearest neighbor algorithm where each center's coordinate points was matched to the other center's coordinate points in a self to other (green-red, red-green) or self to self (green-green, red-red) manner (17). The closest distances were saved, plotted as histograms, and then used to create cumulative curve distributions. STED images of the RCA signals were used to determine the frequency of RCA in relation to the amount of labeled Cot-1 probes. The frequency formula is as follows: $\mathrm{F}=$ (Number of RCA signals)/(Number of Cot-1 probe/2). Frequency was then compared with the 

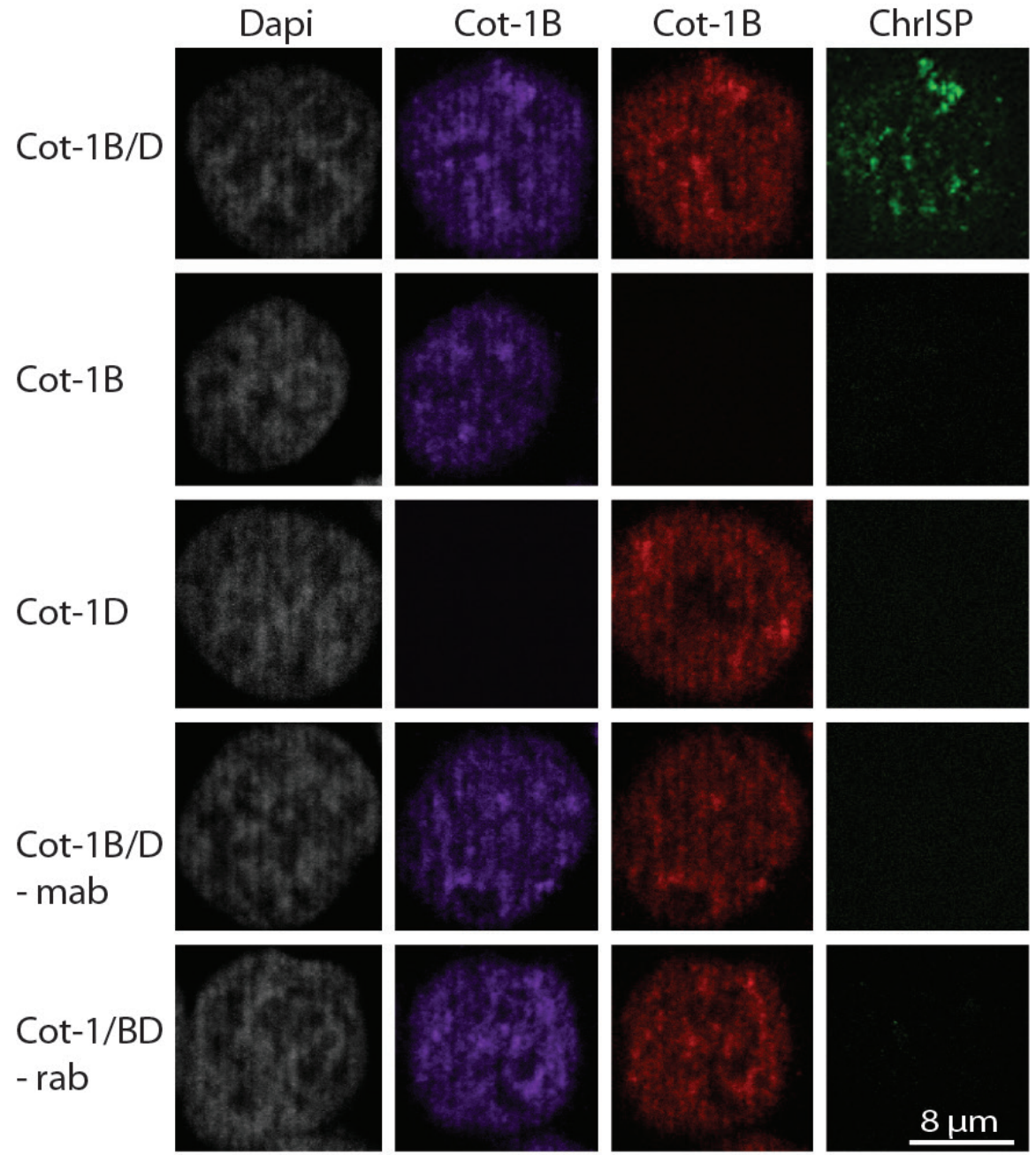

Figure 3. ChrISP analysis of proximity between repeat elements using the green splinter approach. Repeat elements were visualized using biotin- (purple) and digoxigenin-(red) labeled Cot-1 probes, as described in Figure 1. Splinter oligonucleotide ChrISP signal is shown in green. First row shows the positive signals, while the next four rows show the negative controls (i.e. only biotin or digoxigenin probes or only anti-mouse and anti-rabbit $F\left(a b^{\prime}\right)_{2}$ fragment, respectively).

cumulative curve distribution in order to determine the resolution of ChrISP. In total, 15 images from 2 separate samples were acquired and analyzed for the construction of the cumulative curve distribution, and an additional 15 images with RCA were acquired to determine the resolution.

In vitro determination of the sensitivity of the ChrISP method Green splinter oligonucleotides were diluted to different concentrations: $125 \mu \mathrm{M}, 1.25 \mu \mathrm{M}, 0.125 \mu \mathrm{M}$, and 0.0125 $\mu \mathrm{M}$. One $\mu \mathrm{l}$ of each dilution was then added to $99 \mu \mathrm{l}$ of dibutyl phthalate (524980-500Ml, Sigma-Aldrich). The mix was rigorously vortexed for $10 \mathrm{~min}$ to generate the maximum amount of emulsion bubbles. A $5 \mu \mathrm{l}$ aliquot of the mixed solution was then transferred to a coverslip and mounted for microscopy.
Liquid bubbles were scanned and analyzed by the same microscopy and software system used in the ChrISP experiments.

\section{Sequencing and mapping of the} chromosome 11 territory probe The amplified chromosome 11 template was prepared with the TruSeqChIP Sample Preparation kit, Set A (IP-2021012; Illumina, San Diego, CA), and sequencing was done on a Miseq system (SY-410-1003; Illumina). The run produced 5.8 million paired-end reads, of which 4.2 million map to chromosome 11 and 0.7 million map to other chromosomes. Reads were mapped against the human genome (GRCh37. p66, Chr1-22, X, Y, M) using BurrowsWheeler Aligner (BWA; version 0.6.1r104)(bwasw option). The resulting SAM file was processed to identify all reads mapping to repeat regions as defined in the RepeatMasker table using a custom python script (CorrelateToRepeats.py). The RepeatMasker table was downloaded from the UCSC RepeatMasker Human Database. The spacing between these known repeats and their width was analyzed using a custom R-Script (Generate-UCSC-Repeat-DistFigures.R). Repeat regions spaced by $100 \mathrm{bp}$ or less were merged into a single repeat region. The script filters all reads mapping to repeat regions and saves the filtered reads to a separate file. The two SAM files were processed using MACS peak calling software (version 1.4.1). The resulting peak files were analyzed using a custom R script (Make-CT11-Figures.R) to generate distribution of the length of the peaks and the spacing between them. All scripts for mapping of the chromosome 11 probes and the repeat distribution were combined and are included in the Supplementary Material.

\section{Web resources}

RepeatMasker Human Database: http:// hgdownload.soe.ucsc.edu/goldenPath/ hg19/database/rmsk.txt.gz

Human Genome: ftp:/ftp.ensembl.org/ pub/release-66/fasta/homo_sapiens/ dna/

Burrows-Wheeler Aligner (BWA): http:// bio-bwa.sourceforge.net/

$R:$ http://www.r-project.org/

Python: http://www.python.org/

$M A C S$ : http://liulab.dfci.harvard.edu/ MACS/

CT11 Sequence Library: http://www. ncbi.nlm.nih.gov/geo/query/acc. cgi?acc $=$ GSE 42009

\section{Results and discussion}

The proximity between differentially labeled chromatin regions, as visualized by biotin and digoxigenin DNA probes, can be addressed using the ISPLA principle (Figure 1A)(11). The ChrISP application has the potential to unravel chromatin fiber proximities both in cis and in trans. This aim is achieved by employing specific antibodies, which are conjugated with oligo sequences to generate priming and non-priming antibodies. Following addition of splinter oligonucleotides and backbone oligonucleotides, the in situ ligation-mediated formation of a circular DNA serves as an amplification template for Phi29 polymerase (11). The proximity between targeted epitopes is subsequently scored by visualization of rolling circle amplification products using labeled 
oligo probes (11). Figure $1 \mathrm{~B}$ shows that this strategy also works in principle for a chromatin proximity application. Thus, following hybridization of biotin and digoxigenin-labeled Cot-1 DNA enriched in highly repetitive sequences to formaldehyde-fixed HCT116 cells, proximal signals can be readily visualized at regions displaying clustered Cot-1 signals (Figure 1B, Supplementary Figure S1). This signal is specific, as the omission of either the biotin or the digoxigenin-labeled probes did not generate any detectable signal (Figure 1B)

As the interpretation of such data depended on the resolution of the ChrISP signal, we aimed to experimentally determine the distance between the oligo DNA sequences of the proximal antibodies recognized by the splinter oligonucleotide. To this end, we employed STED microscopic analysis of fiber FISH samples (Figure $2 \mathrm{~A}$ and B, Supplementary Figure S2). The strategy was to assess the frequency of the proximities by neighboring Cot-1 signals covering a total of 7-14 million base pairs of the human genome (Figure 2C, Supplementary Figure 3 ) and then compare this information with the proximity frequency generated by ChrISP analysis (see examples in Figure 2B and C). Figure 2D (see also Supplementary Figure S4) shows that the minimal ChrISP resolution is approximately $16 \mathrm{~nm}$, which is very similar to an estimate from another ISPLA application (18).

One disadvantage of using the ISPLA protocol is that the RCA events are sensitive to dextran sulfate (not shown), precluding efficient hybridization protocols. Moreover, the possibility that amplification might be affected by the structural context of the 3-D nuclear architecture is highlighted by the observation that the clusters of Cot-1 ISPLA signals were restricted primarily to the inside rim of the nuclear membrane (Supplementary Movie S1). To deal with this issue, we omitted the amplification step of the ISPLA protocol and instead used a fluorescently labeled (green) splinter oligonucleotide (Figure 1A). The strategy was based on the simultaneous juxtaposition of priming and non-priming oligo DNA sequences to stabilize the formation of the circular DNA. By tweaking the washing conditions, highly specific ChrISP signals could be observed in $100 \%$ of the cells when priming and non-priming antibodies were simultaneously present (Figure 3, Supplementary Figure S5). Moreover, as documented in Supplementary Movie S2, the ChrISP signal can be found in the entire nuclear compartment, lending credibility to the interpretation that, in contrast to the green splinter approach, the RCA step can be sterically hindered in context-specific manners. The difference in hybridization patterns between Figure $1 \mathrm{~B}$ and Figure 3 reflects the more efficient hybridization signal when using dextran sulfate (see Materials and methods).

Although the introduction of the green splinter strategy step reduced the sensitivity of the ISPLA method-about $8 \times 100$ bp probes proximal to each other in a volume of $0.1 \mathrm{~mm}^{3}$ are required for detection (Supplementary Figure S6) it had the huge advantage of providing a continuum of signals, rather than the large blobs that were produced with the original ISPLA protocol. The result was improved visualization of chromatin proximities in relationship to structural hallmarks of the nucleus. This is exemplified in Figure $4 \mathrm{~A}$, which shows clustered interphase chromosome 11 ChrISP signals signifying increased chromatin fiber proximities due to proximity between two different chromosome 11 -specific probes, and that these are enriched at the nuclear membrane of HCT 116 cells (see also Supplementary Figure S7 for an overview). Conversely,
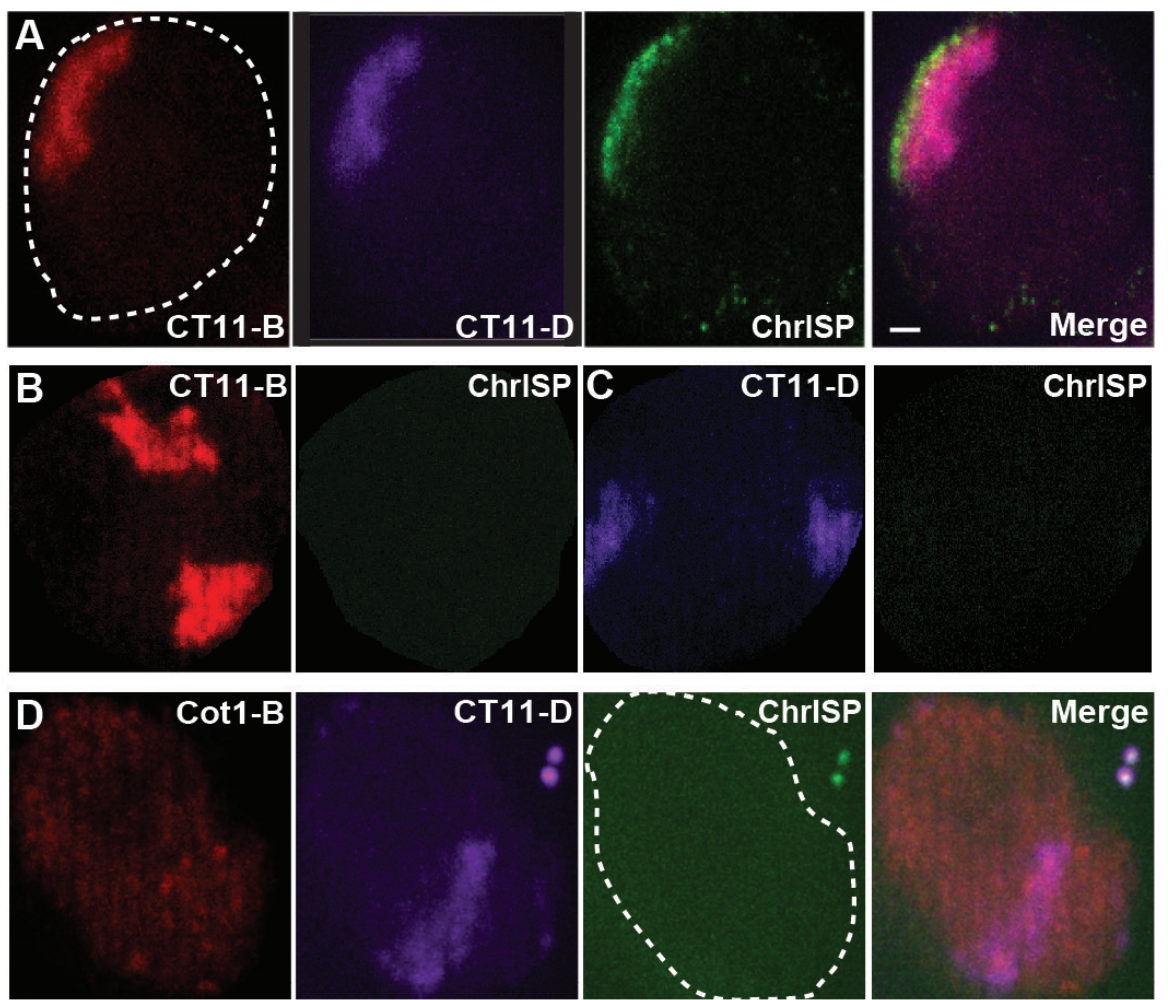

Figure 4. Green splinter ChrISP analysis of the higher order organization of interphase chromosome 11. (A) Chromosome 11 is painted in red and purple following hybridization with biotin- and digoxigenin-labeled chromosome territory probes, respectively. The ChrISP signal is represented by the green splinter approach (see Fig. 1). To control the specificity of the ChrISP signal, either the digoxigenin- (B) or the biotin- (C) labeled probe was omitted. (D) ChrISP analysis of proximity between repeat elements and unique sequences under the same conditions used in (A). Dashed line depicts the boundary of the nucleus. Bar $=2 \mu \mathrm{m}$. 
mentary Figure S9). We conclude that the ChrISP method uniquely visualizes structural features of interphase chromosomes beyond the resolution of the light microscope. Such nuclear membrane-proximal and compacted features may correspond to so-called LOCK domains, which are enriched in the repressive $\mathrm{H} 3 \mathrm{~K} 9 \mathrm{me} 2$ mark (19) (Chen et al., unpublished observation).

The ChrISP technique compares favorably also with other microscopic applications, such as STED, PALM, STORM and super-resolution imaging of FISH. In particular, these applications have limitations not only in the choice of fluorophores but also in offering limited resolution in the 3rd axial dimension. In contrast, as the ChrISP method has a resolution of less than $17 \mathrm{~nm}$ in all three dimensions it provides 3-10-fold improvement of the resolution in the $Z$ plane in comparison with established super-resolution far-field imaging technologies. It is important to note that although the ChrISP signal suffers from the same diffraction limited resolution as other fluorescent signals its presence signifies the close proximity of epitopes.

Using the ChrISP method, we have here documented unique features of chromatin domains in relation to structural hallmarks of the nucleus at the single-cell level in unprecedented detail. To make these applications work, we had to overcome the steric limitations imposed by the nuclear architecture as visualized by the previously established ISPLA technique. By extrapolation, it is now technically possible to directly visualize large-scale changes in chromosome structures as well as chromatin loops and bridges (2) for individual loci in individual cells at high resolution during developmental windows and disease processes.

We describe here a ChrISP technique that enables examination of $3-D$ aspects of higher order chromatin conformations in individual cells at a resolution far beyond that of the confocal microscope. This technique is extremely versatile, allowing visualization of any protein epitope in the proximity of any chromatin domain. The high resolution of the ChrISP technique may further necessitate the development of fixation protocols that preserve the 3D nuclear structure better than formaldehyde. Thus we predict that the ChrISP technique will become very useful in examining the alignment of chromatin domains to structural hallmarks, such as the nuclear membrane and nucleoli, during transcription, RNA splicing, and DNA replication.

\section{Author contributions}

X.C., C.S., S.Y. contributed equally to this work; they accomplished the proof of principle, and implemented, analyzed, and designed the experimental ChrISP work as well as microscopic imaging. X.C. did the DNA fiber experiments and the DNA sequencing. S.Y. designed the green splinter approach, and C.S. assisted with the protocol optimization. A.G. provided the initial idea and proof of principle of ChrISP. D.R. and J.W. performed the STED analysis/calculations of the fiber FISH samples. A.F.W. mapped sequences and performed bioinformatics analyses. N.S helped with the DNA sequencing. R.O. designed the experiments, analyzed the data and wrote the manuscript.

\section{Acknowledgments}

This work was supported by the Swedish Science Research Council, the Swedish Cancer Research Foundation, the Swedish Pediatric Cancer Foundation, the Lundberg Foundation, The KA Wallenberg Foundation, Karolinska Institutet, and ChILL (EU STREP).

\section{Competing interests}

The authors declare no competing interests.

\section{References}

1. Cremer, T., M. Cremer, S. Dietzel, S. Muller, I. Solovei, and S. Fakan. 2006. Chromosome territories--a functional nuclear landscape. Curr. Opin. Cell Biol. 18:307-316.

2. Göndör, A. and R. Ohlsson. 2009 Chromosome crosstalk in three dimensions. Nature 461:212-217.

3. Naumova, N., E. Smith, Y. Zhan, and J. Dekker. 2012. Analysis of long-range chromatin interactions using Chromosome Conformation Capture. Methods. 58:192-203.

4. Göndör, A., C. Rougier, and R. Ohlsson. 2008. High-resolution circular chromosome conformation capture assay. Nat. Protoc. 3:303-313.

5. Solovei, I., J. Walter, M. Cremer, F. Habermann, L. Schermelleh, and T. Cremer. 2002. Three-dimensionally preserved nuclei, p. 119-157. In B. Beatty, S. Mai, and J. Squire (Eds.), FISH: A Practical Approach. Oxford University Press, Oxford.

6. Wildanger, D., E. Rittweger, L. Kastrup, and S.W. Hell. 2008. STED microscopy with a supercontinuum laser source. Opt. Express 16:9614-9621.

7. Markaki, Y., D. Smeets, S. Fiedler, V.J. Schmid, L. Schermelleh, T. Cremer, and $M$. Cremer. 2012. The potential of 3D-FISH and super-resolution structured illumination microscopy for studies of 3D nuclear architecture: 3D structured illumination microscopy of defined chromosomal structures visualized by $3 \mathrm{D}$ (immuno)-FISH opens new perspectives for studies of nuclear architecture. BioEssays 34:412-426.

8. Wildanger, D., R. Medda, L. Kastrup, and S.W. Hell. 2009. A compact STED microscope providing $3 \mathrm{D}$ nanoscale resolution. J. Microsc. 236:35-43.

9. Pujadas, E. and A.P. Feinberg. 2012. Regulated noise in the epigenetic landscape of development and disease. Cell 148:1123-1131.

10. Nagano, T., Y. Lubling, T.J. Stevens, S. Schoenfelder, E. Yaffe, W. Dean, E.D. Laue, A. Tanay, and P. Fraser. 2013. Single-cell Hi-C reveals cell-to-cell variability in chromosome structure. Nature 502:59-64.

11. Söderberg, O., M. Gullberg, M. Jarvius, K. Ridderstrale, K.J. Leuchowius, J. Jarvius, K. Wester, P. Hydbring, et al. 2006. Direct observation of individual endogenous protein complexes in situ by proximity ligation. Nat. Methods 3:995-1000.

12. Cremer, M., F. Grasser, C. Lanctot, S. Muller, M. Neusser, R. Zinner, I. Solovei, and T. Cremer. 2008. Multicolor 3D fluorescence in situ hybridization for imaging interphase chromosomes. Methods Mol. Biol. 463:205239.

13. Sandhu, K.S., C. Shi, M. Sjolinder, Z. Zhao, A. Gondor, L. Liu, V.K. Tiwari, S. Guibert, et al. 2009. Nonallelic transvection of multiple imprinted loci is organized by the H19 imprinting control region during germline development. Genes Dev. 23:2598-2603.

14. Courbet, S., S. Gay, N. Arnoult, G. Wronka, M. Anglana, O. Brison, and M. Debatisse. 2008 Replication fork movement sets chromatin loop size and origin choice in mammalian cells. Nature 455:557-560.

15. Neumann, D., J. Buckers, L. Kastrup, S.W. Hell, and S. Jakobs. 2010. Two-color STED microscopy reveals different degrees of colocalization between hexokinase-I and the three human VDAC isoforms. PMC Biophysics 3:4.

16. Richardsson, W. 1972. Bayesian-Based Iterative Method of Image Restoration. J. Opt. Soc. Am. 62:55-59.

17. Blom, H., D. Ronnlund, L. Scott, Z. Spicarova, V. Rantanen, J. Widengren, A. Aperia, and H. Brismar. 2012. Nearest neighbor analysis of dopamine $\mathrm{D} 1$ receptors and $\mathrm{Na}(+)-\mathrm{K}(+)$ ATPases in dendritic spines dissected by STED microscopy. Microsc. Res. Tech. 75:220-228.

18. Trifilieff, P., M.L. Rives, E. Urizar, R.A. Piskorowski, H.D. Vishwasrao, J. Castrillon, C. Schmauss, M. Slattman, et al. 2011. Detection of antigen interactions ex vivo by proximity ligation assay: endogenous dopamine $\mathrm{D} 2$-adenosine $\mathrm{A} 2 \mathrm{~A}$ receptor complexes in the striatum. BioTechniques 51:111-118.

19. Wen, B., H. Wu, Y. Shinkai, R.A. Irizarry, and A.P. Feinberg. 2009. Large histone H3 lysine 9 dimethylated chromatin blocks distinguish differentiated from embryonic stem cells. Nat. Genet. 41:246-250.

Received 24 October 2013; accepted 16 January 2014.

Address correspondence to Rolf Ohlsson, Department of Microbiology, Tumor and Cell Biology, Karolinska Institutet, Stockholm, Sweden. E-mail: rolf.ohlsson@ki.se

To purchase reprints of this article, contact: biotechniques@fosterprinting.com 